\title{
Comparative FISH-mapping of TTAGG telomeric sequences to the chromosomes of leafcutter ants (Formicidae, Myrmicinae): is the insect canonical sequence conserved?
}

\author{
Carini Picardi Morais de Castro',2, Danon Clemes Cardoso', \\ Ricardo Micolino ${ }^{3}$, Maykon Passos Cristiano'
}

I Departamento de Biodiversidade, Evolução e Meio Ambiente, Universidade Federal de Ouro Preto (UFOP), MG, Brazil 2 Departament de Genètica, Facultat de Biologia, Universitat de Barcelona (UB), Barcelona, Spain 3 Programa de Pós-graduação em Genética, Universidade Federal do Paraná (UFPR), Curitiba, PR, Brazil

Corresponding author: Danon Clemes Cardoso (danon@ufop.edu.br);

Maykon Passos Cristiano (maykon@ufop.edu.br)

Academic editor: Marco Gebiola | Received 30 March 2020 | Accepted 30 May 2020 | Published 14 August 2020

http://zoobank.org/02AEB841-9BD3-4C95-B03F-36B062B6D1B2

Citation: de Castro CPM, Cardoso DC, Micolino R, Cristiano MP (2020) Comparative FISH-mapping of TTAGG telomeric sequences to the chromosomes of leafcutter ants (Formicidae, Myrmicinae): is the insect canonical sequence conserved?. Comparative Cytogenetics 14(3): 369-385. https://doi.org/10.3897/CompCytogen.v14i3.52726

\begin{abstract}
Telomeric sequences are conserved across species. The most common sequence reported among insects is (TTAGG), but its universal occurrence is not a consensus because other canonical motifs have been reported. In the present study, we used fluorescence in situ hybridization (FISH) using telomeric probes with (TTAGG) ${ }_{6}$ repeats to describe the telomere composition of leafcutter ants. We performed the molecular cytogenetic characterization of six Acromyrmex Mayr, 1865 and one Atta Fabricius, 1804 species (Acromyrmex ambiguus (Emery, 1888), Ac. crassispinus (Forel, 1909), Ac. lundii (Guérin-Mèneville, 1838), Ac. nigrosetosus (Forel, 1908), Ac. rugosus (Smith, 1858), Ac. subterraneus subterraneus (Forel, 1893), and Atta sexdens (Linnaeus, 1758)) and described it using a karyomorphometric approach on their chromosomes. The diploid chromosome number $2 \mathrm{n}=38$ was found in all Acromyrmex species, and the karyotypic formulas were as follows: Ac. ambiguus $2 \mathrm{~K}=14 \mathrm{M}+12 \mathrm{SM}+8 \mathrm{ST}+4 \mathrm{~A}$, Ac. crassispinus $2 \mathrm{~K}=12 \mathrm{M}+20 \mathrm{SM}+4 \mathrm{ST}+2 \mathrm{~A}, A c$. lundii $2 \mathrm{~K}=10 \mathrm{M}+14 \mathrm{SM}+10 \mathrm{ST}+4 \mathrm{~A}$, Ac. nigrosetosus $2 \mathrm{~K}=12 \mathrm{M}+14 \mathrm{SM}+10 \mathrm{ST}+2 \mathrm{~A}$, and Ac. subterraneus subterraneus $2 \mathrm{~K}=14 \mathrm{M}+18 \mathrm{SM}+4 \mathrm{ST}+2 \mathrm{~A}$. The exact karyotypic formula was not established for Ac. rugosus. FISH analyses revealed the telomeric regions in all the chromosomes of the species studied in the present work were marked by the (TTAGG) 6 sequence. These results reinforce the premise that Formicidae presents high homology between their genera for the presence of the canonical sequence (TTAGG) ${ }_{n}$.
\end{abstract}

Copyright C.P.M. de Castro et al. This is an open access article distributed under the terms of the Creative Commons Attribution License (CC BY 4.0), which permits unrestricted use, distribution, and reproduction in any medium, provided the original author and source are credited. 


\section{Keywords}

evolution, FISH, insects, leafcutter ants, telomere

\section{Introduction}

Cytogenetic studies have been performed on more than 750 ant species, most of which describe only the chromosome number and morphology (Lorite and Palomeque 2010; Cardoso et al. 2018a). However, the cytogenetic information available so far represents less than $5 \%$ of the known ant species. Formicidae is very diverse with respect to both karyotype and species. The subfamily Myrmicinae comprises more than 400 species with established karyotypes and haploid chromosome counts varying from $\mathrm{n}=2$ to $\mathrm{n}=35$ (Cardoso et al. 2018a). Myrmicinae includes the leafcutter ants in the genera Atta Fabricius, 1804 to Acromyrmex Mayr, 1865 that occur exclusively in the Neotropical region and are extremely important herbivores in the habitats that they occupy. They cut thousands of fresh plant pieces that are transported to nests and this habit is essential for cycling soil nutrients, mainly carbon (Farji-Brener and Ghermandi 2008). In some cases, Atta and Acromyrmex are considered agricultural pests due to the economic damages caused by their habit of cutting green leaves; therefore, most studies usually focus on their ecology, geographic distribution, and population control (Loeck et al. 2003). However, both genera need a systematic revision and a complete picture of their unclear phylogenetic relationships.

The genus Atta includes 17 species (Bolton 2020), of which five have an established karyotype. All species present the diploid chromosome number, $2 \mathrm{n}=22$, and the karyotype formula, $2 \mathrm{~K}=18 \mathrm{M}+4 \mathrm{~A}$, except for Atta robusta Borgmeier, 1939, which has the formula $2 \mathrm{~K}=18 \mathrm{M}+2 \mathrm{SM}+2 \mathrm{ST}$ (reviewed in Cardoso et al. 2018a). The genus Acromyrmex has 34 species and 29 subspecies that are currently recognized (Bolton 2020), it has the diploid chromosome number $2 \mathrm{n}=38$ and its karyotype formula is variable (Barros et al. 2016; reviewed in Cardoso et al. 2018a). The exceptions in the genus are Acromyrmex ameliae de Souza, Soares \& Della Lucia, 2007, that has $2 \mathrm{n}=36$ (reviewed in Cardoso et al. 2018a) and Acromyrmex striatus (Roger, 1863) which presents $2 \mathrm{n}=$ 22 (Cristiano et al. 2013). The only species whose karyotype has been characterized by morphometric analyses so far is Ac. striatus (Cristiano et al. 2013). Such chromosomal features are essential for understanding chromosomal variants and the possible genetic barriers among phylogenetic groups (Cardoso et al. 2018b). Ac. striatus is a key species within the evolutionary history of leafcutter ants because molecular analyses and its karyotype establishment resulted in reclassification of Acromyrmex as paraphyletic. Although Ac. striatus shares the characteristics of both Acromyrmex and Atta, it presents peculiarities such as its karyotype formula $2 \mathrm{~K}=20 \mathrm{M}+2 \mathrm{SM}$, indicating that Ac. striatus should be better classified as a genus distinct from its sibling leafcutter ants (Cristiano et al. 2013).

Karyo-evolutionary pathways can be accurately established from molecular analyses by means of fluorescence in situ hybridization (FISH), a chromosomal mapping 
technique that allows identification of specific genomic regions through hybridization of fluorescent probes to the genetic material (Speicher and Carter 2005). Probe origin may range from single or repetitive sequences to large genomic sequences and probes from telomeric repeating regions are commonly applied in cytogenetic studies (Micolino et al. 2019a, b, 2020; Travenzoli et al. 2019). Telomeres are located at terminal portions of chromosomes, which are enriched with repetitive bases of adenine $(A)$, guanine $(G)$, and thymine $(T)$ and the number of repeated base pairs can be extremely conserved among some taxonomic groups (Blackburn 1991; Zakian 1995). Four different telomeric sequences have been identified in Insecta, but the pentanucleotide region (TTAGG) is present in most insects (Okazaki et al. 1993; Sahara et al. 1999). Thus, it is presumed that this motif is derived from a common ancestor and is therefore homologous among the class orders (Vítková et al. 2005). However, many Hymenoptera families do not present the sequence in their chromosomes (Menezes et al. 2017), whereas some families have several species that show telomeric regions marked by the presence of $\left(\right.$ TTAGG) ${ }_{n}$ or the vertebrate canonical repetition (TTAGGG) , as in the case of Apidae (Sahara et al. 1999), Formicidae (Okazaki et al. 1993; Meyne et al. 1995; Lorite et al. 2002; Wurm et al. 2011) and Tenthredinidae, which has two species presenting the insect canonical sequence (Gokhman and Kuznetsova 2018).

The pentanucleotide sequence has apparently evolved from the canonical sequence (TTAGGG) ${ }_{\mathrm{n}}$ and has changed during insect diversification. This is supported by families that show the presence of (TTAGGG) and also by genera which present a different telomeric sequence such as (TCAGG) $)_{n}$, which is observed in some Coleoptera families (Kuznetsova et al. 2019). The differences in telomeric sequences within the class Insecta can be explained by biological mechanisms that preserve the telomere integrity. Telomerase is the enzyme responsible for maintaining repetitive sequences on telomeres; however, many alternative telomeraseindependent mechanisms also act in telomere conservation. In this manner, the (TTAGG) ${ }_{\mathrm{n}}$ sequence has been lost and recovered several times during the evolution of insects (Kuznetsova et al. 2019).

Other than chromosome number, not much cytogenetic information is available regarding leafcutter ants, and FISH analyses involving telomeric probes are available only for Ac. striatus (Pereira et al. 2018). Further, the distribution of canonical repeats and telomerase systems is still an open question among insects (Kuznetsova et al. 2019). Thus, in the present study, we analyzed the homology between the telomeric regions of leafcutter ant species Ac. ambiguus (Emery, 1888), Ac. crassispinus (Forel, 1909), Ac. lundii (Guérin-Mèneville, 1838), Ac. nigrosetosus (Forel, 1908), Ac. rugosus (Smith, 1858), Ac. subterraneus subterraneus (Forel, 1893), and Atta sexdens (Linnaeus, 1758) by FISH chromosome mapping using the (TTAGG) ${ }_{6}$ probe. We aimed to accumulate evolutionary evidence for the presence of an insect canonical telomere motif on the chromosomes of leafcutter ants. We further performed a detailed karyomorphometric analysis to establish karyotypes and classify chromosome, and described two new chromosome counts. 


\section{Material and methods}

\section{Chromosome preparation and fluorescence in situ hybridization (FISH)}

The ant colonies were collected from different Brazilian states in 2018. Acromyrmex ambiguus was collected from Ilha Comprida - SP (244' $\left.28^{\circ} \mathrm{S}, 47^{\circ} 32^{\prime} 24^{\prime \prime W}\right)$; the species Ac. crassispinus (Ouro Preto - 20 $\left.0^{\circ} 17^{\prime} 15^{\prime \prime} \mathrm{S}, 3^{\circ} 30^{\prime} 29^{\prime \prime} \mathrm{W}\right)$, Ac. rugosus (Marliéria -

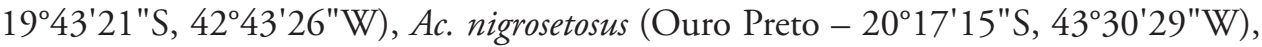
Ac. subterraneus subterraneus (Viçosa - 20 $0^{\circ} 48^{\prime} 35.5^{\prime \prime} \mathrm{S}, 42^{\circ} 51^{\prime} 31.07^{\prime \prime} \mathrm{W}$ ), and At. sexdens (Marliéria - 19 $43^{\prime} 21$ 'S, 42\%43'26"W) were collected in Minas Gerais - MG; Ac. lundii was collected in Dom Pedrito - RS (30 $58^{\prime} 5^{\prime \prime}$ S, 5440'23"W). The nests were kept at the Laboratório de Genética Evolutiva e de Populaçóes of the Universidade Federal de Ouro Preto. The brain ganglia of post-defective larvae were extracted in hypotonic solution of colchicine $(0.005 \%)$, as described by Imai et al. (1988) with modifications described by Cardoso et al. (2012), to obtain the metaphasic chromosomes.

FISH experiments were performed as described by Micolino et al. (2019a). The (TTAGG) ${ }_{6}$ motif was directly labeled with $\mathrm{Cy} 3$ at the 5 ' terminal (Sigma, St. Louis, $\mathrm{MO}, \mathrm{USA}$ ). Briefly, slides were submitted to RNA degradation for $1 \mathrm{~h}$ in a humid chamber at $37{ }^{\circ} \mathrm{C}$, were washed in $2 \times$ SSC, and treated with $0.005 \%$ pepsin for 10 min. After washing in $1 \times$ PBS, the slides were fixed with $10 \%$ formaldehyde for 10 min. Another wash in $1 \times$ PBS was performed and then, the slides were dehydrated in an alcohol series. Chromosomal denaturation was promoted by adding $70 \%$ formamide at $75{ }^{\circ} \mathrm{C}$ for $5 \mathrm{~min}$. Another alcohol dehydration series was performed before adding $2 \mu \mathrm{L}$ of the (TTAGG) ${ }_{6}$ probe and $18 \mu \mathrm{L}$ of HybMix to each slide in the dark. The slides were incubated overnight in a humid chamber at $37^{\circ} \mathrm{C}$. Finally, the slides were washed in $2 \times$ SSC solution, $1 \times$ SSC, $4 \times$ SSC Tween (during 5 min in each solution), and then rapidly in $1 \times$ PBS. Dehydration was performed in an alcohol series and DAPI was added as a counterstain. To select 10 metaphases with chromosomal integrity and evident probe marking, the slides were visualized on a Zeiss Axio Imager Z2 fluorescence microscope coupled with an image capture system and the resulting images were further edited using Adobe Photoshop CC Software.

\section{Karyomorphometry}

The slides were stained with a $4 \%$ Giemsa solution and visualized on a Zeiss Axio Imager Z2 microscope with image capture. For each species, we selected 10 metaphases with chromosomal integrity, evident centromeres and no overlapping. Karyomorphometry and chromosomal classification were performed as described by Cristiano et al. (2017). The chromosomes were measured using Image-Pro Plus (Media Cybernetics, Rockville, MD) and some chromosome characteristics were evaluated. For each chromosome, we measured the total length (TL) end-to-end, short arm (S), and long arm (L) sizes calculated by the distance between the arm end and centromeric region. The 
karyotype length (KL) was calculated by summing the total length of all chromosomes. The relative size (RL) of each chromosome was calculated in relation to the total size of all chromosomes $(\mathrm{TL} \times 100 / \Sigma \mathrm{TL})$. The ratio $(\mathrm{r})$ between the length of the long arm and short arm $(\mathrm{r}=\mathrm{L} / \mathrm{S})$ was calculated to classify the chromosomes as described by Levan et al. (1964) with modifications reported by Crozier (1970).

\section{Results}

The typical chromosome number of Acromyrmex $(2 \mathrm{n}=38)$ was found in all species of the genus analyzed in the present work. The karyotype of Ac. lundii and Ac. nigrosetosus were described for the first time and, that of $A c$. ambiguus was described for the first time from a Brazilian population. The two largest chromosomal pairs were the first subtelocentric and the first metacentric. The karyotype formula was variable (see below) and in Ac. crassispinus, Ac. lundii, Ac. nigrosetosus, and Ac. subterraneus subterraneus, most chromosomes presented an $\mathrm{r}$ ratio between 1.67 and 3.00; therefore, these were classified as submetacentric. The chromosomal classification of Ac. ambiguus was different from that of other species, as it mainly presents metacentric chromosomes. Ac. ambiguus has the karyotype formula $2 \mathrm{~K}=14 \mathrm{M}+12 \mathrm{SM}+8 \mathrm{ST}+4 \mathrm{~A}$ (Figure 1, Table 1). Ac. crassispinus presented $2 \mathrm{~K}=12 \mathrm{M}+20 \mathrm{SM}+4 \mathrm{ST}+2 \mathrm{~A}$ (Figure 2, Table 2) and its chromosomes are larger when compared to other species. Ac. lundii has the karyotype formula $2 \mathrm{~K}=10 \mathrm{M}+14 \mathrm{SM}+10 \mathrm{ST}+4 \mathrm{~A}$ (Figure 3, Table 3). Ac. nigrosetosus presented $2 \mathrm{~K}=12 \mathrm{M}+14 \mathrm{SM}+10 \mathrm{ST}+2 \mathrm{~A}$ and its chromosomes seem smaller than those of the other species (Figure 4, Table 4). Ac. subterraneus subterraneus has $2 \mathrm{~K}=14 \mathrm{M}+$

A

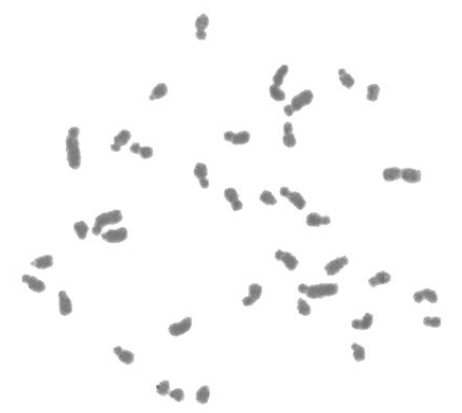

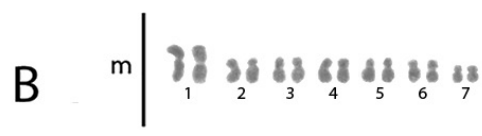
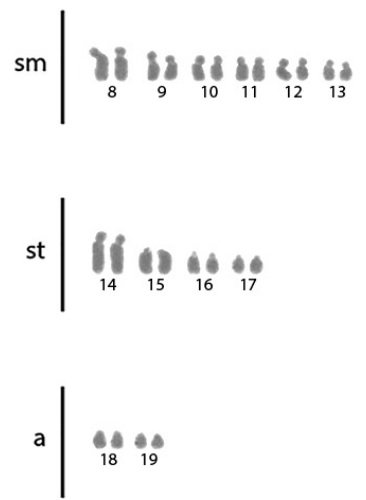

Figure I. Conventional staining of mitotic cells of Acromyrmex ambiguus $\mathbf{A}$ the metaphase and $\mathbf{B}$ diploid karyotype with $2 \mathrm{n}=38$. Scale bar: $5 \mu \mathrm{m}$. 
Table I. Karyomorphometric analyses of the chromosomes of Acromyrmex ambiguus.

\begin{tabular}{|c|c|c|c|c|c|c|}
\hline Chromosomes & TL & $\mathbf{L}$ & $S$ & RL & $\mathbf{r}$ & Classification \\
\hline 1 & $5.13 \pm 1.90$ & $2.79 \pm 1.03$ & $2.34 \pm 0.89$ & $4.41 \pm 0.39$ & $1.20 \pm 0.14$ & Metacentric \\
\hline 1 & $4.85 \pm 1.87$ & $2.60 \pm 1.01$ & $2.26 \pm 0.89$ & $4.17 \pm 0.46$ & $1.15 \pm 0.15$ & Metacentric \\
\hline 2 & $3.35 \pm 1.11$ & $1.96 \pm 0.68$ & $1.39 \pm 0.43$ & $2.91 \pm 0.14$ & $1.40 \pm 0.14$ & Metacentric \\
\hline 2 & $3.18 \pm 0.98$ & $1.87 \pm 0.58$ & $1.31 \pm 0.41$ & $2.78 \pm 0.05$ & $1.43 \pm 0.14$ & Metacentric \\
\hline 3 & $3.11 \pm 0.94$ & $1.83 \pm 0.59$ & $1.29 \pm 0.36$ & $2.72 \pm 0.07$ & $1.41 \pm 0.10$ & Metacentric \\
\hline 3 & $3.08 \pm 0.93$ & $1.80 \pm 0.58$ & $1.28 \pm 0.36$ & $2.69 \pm 0.07$ & $1.40 \pm 0.10$ & Metacentric \\
\hline 4 & $3.01 \pm 0.92$ & $1.76 \pm 0.57$ & $1.25 \pm 0.36$ & $2.63 \pm 0.06$ & $1.40 \pm 0.13$ & Metacentric \\
\hline 4 & $2.92 \pm 0.89$ & $1.77 \pm 0.54$ & $1.15 \pm 0.35$ & $2.55 \pm 0.09$ & $1.54 \pm 0.10$ & Metacentric \\
\hline 5 & $2.86 \pm 0.84$ & $1.71 \pm 0.52$ & $1.15 \pm 0.33$ & $2.50 \pm 0.08$ & $1.48 \pm 0.13$ & Metacentric \\
\hline 5 & $2.76 \pm 0.77$ & $1.62 \pm 0.47$ & $1.14 \pm 0.32$ & $2.43 \pm 0.15$ & $1.43 \pm 0.18$ & Metacentric \\
\hline 6 & $2.65 \pm 0.69$ & $1.61 \pm 0.44$ & $1.04 \pm 0.25$ & $2.35 \pm 0.18$ & $1.54 \pm 0.12$ & Metacentric \\
\hline 6 & $2.56 \pm 0.66$ & $1.47 \pm 0.36$ & $1.09 \pm 0.33$ & $2.27 \pm 0.21$ & $1.39 \pm 0.18$ & Metacentric \\
\hline 7 & $2.28 \pm 0.62$ & $1.37 \pm 0.40$ & $0.90 \pm 0.23$ & $2.02 \pm 0.22$ & $1.51 \pm 0.10$ & Metacentric \\
\hline 7 & $2.13 \pm 0.55$ & $1.22 \pm 0.31$ & $0.90 \pm 0.26$ & $1.89 \pm 0.17$ & $1.37 \pm 0.18$ & Metacentric \\
\hline 8 & $4.35 \pm 1.37$ & $3.17 \pm 1.02$ & $1.18 \pm 0.36$ & $3.79 \pm 0.18$ & $2.68 \pm 0.27$ & Submetacentric \\
\hline 8 & $4.11 \pm 1.27$ & $3.01 \pm 0.95$ & $1.10 \pm 0.33$ & $3.59 \pm 0.16$ & $2.73 \pm 0.19$ & Submetacentric \\
\hline 9 & $3.35 \pm 0.99$ & $2.36 \pm 0.77$ & $0.98 \pm 0.26$ & $2.94 \pm 0.13$ & $2.40 \pm 0.41$ & Submetacentric \\
\hline 9 & $3.15 \pm 0.94$ & $2.21 \pm 0.76$ & $0.94 \pm 0.21$ & $2.76 \pm 0.08$ & $2.33 \pm 0.44$ & Submetacentric \\
\hline 10 & $3.11 \pm 0.91$ & $2.15 \pm 0.68$ & $0.95 \pm 0.25$ & $2.73 \pm 0.09$ & $2.25 \pm 0.32$ & Submetacentric \\
\hline 10 & $3.07 \pm 0.92$ & $2.17 \pm 0.73$ & $0.90 \pm 0.22$ & $2.69 \pm 0.07$ & $2.41 \pm 0.44$ & Submetacentric \\
\hline 11 & $2.98 \pm 0.91$ & $2.08 \pm 0.66$ & $0.90 \pm 0.26$ & $2.60 \pm 0.08$ & $2.33 \pm 0.28$ & Submetacentric \\
\hline 11 & $2.90 \pm 0.86$ & $2.00 \pm 0.63$ & $0.90 \pm 0.24$ & $2.54 \pm 0.07$ & $2.20 \pm 0.22$ & Submetacentric \\
\hline 12 & $2.70 \pm 0.68$ & $1.77 \pm 0.58$ & $0.93 \pm 0.20$ & $2.40 \pm 0.22$ & $2.08 \pm 0.28$ & Submetacentric \\
\hline 12 & $2.57 \pm 0.67$ & $1.76 \pm 0.45$ & $0.81 \pm 0.23$ & $2.29 \pm 0.24$ & $2.21 \pm 0.22$ & Submetacentric \\
\hline 13 & $2.47 \pm 0.66$ & $1.73 \pm 0.47$ & $0.75 \pm 0.20$ & $2.19 \pm 0.23$ & $2.33 \pm 0.28$ & Submetacentric \\
\hline 13 & $2.19 \pm 0.51$ & $1.49 \pm 0.39$ & $0.70 \pm 0.14$ & $1.96 \pm 0.25$ & $2.10 \pm 0.26$ & Submetacentric \\
\hline 14 & $5.22 \pm 1.84$ & $4.15 \pm 1.60$ & $1.07 \pm 0.30$ & $4.50 \pm 0.32$ & $3.86 \pm 0.97$ & Subtelocentric \\
\hline 14 & $4.76 \pm 1.56$ & $3.79 \pm 1.33$ & $0.97 \pm 0.26$ & $4.14 \pm 0.19$ & $3.86 \pm 0.66$ & Subtelocentric \\
\hline 15 & $3.23 \pm 1.24$ & $2.61 \pm 1.03$ & $0.62 \pm 0.22$ & $2.77 \pm 0.29$ & $4.13 \pm 0.62$ & Subtelocentric \\
\hline 15 & $2.99 \pm 1.15$ & $2.35 \pm 0.93$ & $0.64 \pm 0.25$ & $2.56 \pm 0.31$ & $3.68 \pm 0.65$ & Subtelocentric \\
\hline 16 & $2.69 \pm 1.05$ & $2.15 \pm 0.88$ & $0.54 \pm 0.19$ & $2.29 \pm 0.25$ & $3.98 \pm 0.60$ & Subtelocentric \\
\hline 16 & $2.55 \pm 0.96$ & $1.98 \pm 0.76$ & $0.57 \pm 0.21$ & $2.18 \pm 0.20$ & $3.49 \pm 0.45$ & Subtelocentric \\
\hline 17 & $2.39 \pm 0.87$ & $1.91 \pm 0.73$ & $0.48 \pm 0.17$ & $2.05 \pm 0.16$ & $4.00 \pm 0.93$ & Subtelocentric \\
\hline 17 & $2.21 \pm 0.76$ & $1.74 \pm 0.58$ & $0.48 \pm 0.20$ & $1.91 \pm 0.14$ & $3.93 \pm 1.11$ & Subtelocentric \\
\hline 18 & $2.03 \pm 0.48$ & $1.83 \pm 0.43$ & $0.20 \pm 0.06$ & $1.82 \pm 0.22$ & $9.14 \pm 1.41$ & Acrocentric \\
\hline 18 & $1.95 \pm 0.47$ & $1.73 \pm 0.41$ & $0.22 \pm 0.07$ & $1.74 \pm 0.20$ & $8.27 \pm 0.99$ & Acrocentric \\
\hline 19 & $1.85 \pm 0.43$ & $1.66 \pm 0.39$ & $0.19 \pm 0.04$ & $1.66 \pm 0.19$ & $9.02 \pm 0.91$ & Acrocentric \\
\hline 19 & $1.75 \pm 0.42$ & $1.57 \pm 0.39$ & $0.18 \pm 0.03$ & $1.56 \pm 0.19$ & $8.79 \pm 1.21$ & Acrocentric \\
\hline KL & 114.44 & & & & & \\
\hline
\end{tabular}

TL: total length; L: long arm length; S: short arm length; RL: relative length; r: arm ratio, KL: karyotype length.

A

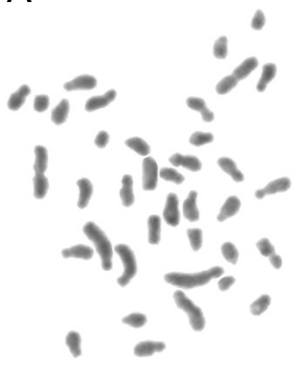

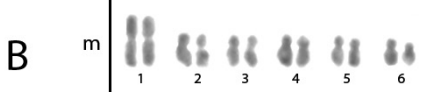
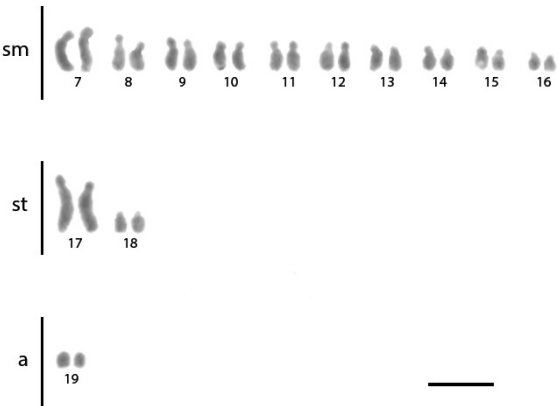

Figure 2. Conventional staining of mitotic cells of Acromyrmex crassispinus $\mathbf{A}$ the metaphase and $\mathbf{B}$ diploid karyotype with $2 \mathrm{n}=38$. Scale bar: $5 \mu \mathrm{m}$. 
Table 2. Karyomorphometric analyses of the chromosomes of Acromyrmex crassispinus.

\begin{tabular}{|c|c|c|c|c|c|c|}
\hline Chromosomes & TL & $\mathbf{L}$ & $S$ & RL & $\mathbf{r}$ & Classification \\
\hline 1 & $5.84 \pm 0.93$ & $3.10 \pm 0.51$ & $2.74 \pm 0.47$ & $4.35 \pm 0.22$ & $1.14 \pm 0.12$ & Metacentric \\
\hline 1 & $5.66 \pm 0.93$ & $3.04 \pm 0.44$ & $2.62 \pm 0.50$ & $4.21 \pm 0.21$ & $1.17 \pm 0.08$ & Metacentric \\
\hline 2 & $3.96 \pm 0.76$ & $2.36 \pm 0.53$ & $1.61 \pm 0.28$ & $2.94 \pm 0.20$ & $1.47 \pm 0.20$ & Metacentric \\
\hline 2 & $3.74 \pm 0.60$ & $2.24 \pm 0.32$ & $1.50 \pm 0.31$ & $2.79 \pm 0.10$ & $1.51 \pm 0.14$ & Metacentric \\
\hline 3 & $3.63 \pm 0.56$ & $2.10 \pm 0.24$ & $1.45 \pm 0.20$ & $2.71 \pm 0.10$ & $1.46 \pm 0.15$ & Metacentric \\
\hline 3 & $3.58 \pm 0.56$ & $1.98 \pm 0.31$ & $1.60 \pm 0.29$ & $2.67 \pm 0.10$ & $1.25 \pm 0.14$ & Metacentric \\
\hline 4 & $3.48 \pm 0.50$ & $2.04 \pm 0.34$ & $1.43 \pm 0.19$ & $2.60 \pm 0.08$ & $1.43 \pm 0.13$ & Metacentric \\
\hline 4 & $3.38 \pm 0.48$ & $2.01 \pm 0.27$ & $1.37 \pm 0.23$ & $2.53 \pm 0.12$ & $1.49 \pm 0.14$ & Metacentric \\
\hline 5 & $3.23 \pm 0.46$ & $1.94 \pm 0.30$ & $1.30 \pm 0.18$ & $2.42 \pm 0.15$ & $1.50 \pm 0.10$ & Metacentric \\
\hline 5 & $3.11 \pm 0.49$ & $1.85 \pm 0.33$ & $1.27 \pm 0.18$ & $2.33 \pm 0.18$ & $1.45 \pm 0.13$ & Metacentric \\
\hline 6 & $2.94 \pm 0.53$ & $1.63 \pm 0.42$ & $1.14 \pm 0.27$ & $2.19 \pm 0.21$ & $1.43 \pm 0.16$ & Metacentric \\
\hline 6 & $3.01 \pm 1.11$ & $1.86 \pm 0.89$ & $1.14 \pm 0.31$ & $2.21 \pm 0.57$ & $1.60 \pm 0.45$ & Metacentric \\
\hline 7 & $5.02 \pm 0.83$ & $3.57 \pm 0.54$ & $1.45 \pm 0.37$ & $3.74 \pm 0.12$ & $2.53 \pm 0.42$ & Submetacentric \\
\hline 7 & $4.72 \pm 0.86$ & $3.22 \pm 0.90$ & $1.50 \pm 0.45$ & $3.51 \pm 0.24$ & $2.49 \pm 0.29$ & Submetacentric \\
\hline 8 & $3.99 \pm 0.58$ & $2.70 \pm 0.44$ & $1.29 \pm 0.23$ & $2.98 \pm 0.12$ & $2.14 \pm 0.39$ & Submetacentric \\
\hline 8 & $3.85 \pm 0.59$ & $2.66 \pm 0.40$ & $1.20 \pm 0.23$ & $2.87 \pm 0.08$ & $2.25 \pm 0.34$ & Submetacentric \\
\hline 9 & $3.78 \pm 0.57$ & $2.65 \pm 0.37$ & $1.13 \pm 0.24$ & $2.82 \pm 0.08$ & $2.39 \pm 0.34$ & Submetacentric \\
\hline 9 & $3.70 \pm 0.60$ & $2.56 \pm 0.50$ & $1.14 \pm 0.19$ & $2.75 \pm 0.08$ & $2.29 \pm 0.45$ & Submetacentric \\
\hline 10 & $3.64 \pm 0.57$ & $2.51 \pm 0.45$ & $1.13 \pm 0.20$ & $2.71 \pm 0.07$ & $2.25 \pm 0.41$ & Submetacentric \\
\hline 10 & $3.56 \pm 0.52$ & $2.43 \pm 0.35$ & $1.12 \pm 0.22$ & $2.65 \pm 0.05$ & $2.20 \pm 0.33$ & Submetacentric \\
\hline 11 & $3.48 \pm 0.48$ & $2.41 \pm 0.36$ & $1.07 \pm 0.17$ & $2.60 \pm 0.08$ & $2.27 \pm 0.34$ & Submetacentric \\
\hline 11 & $3.39 \pm 0.50$ & $2.32 \pm 0.40$ & $1.07 \pm 0.18$ & $2.53 \pm 0.07$ & $2.19 \pm 0.42$ & Submetacentric \\
\hline 12 & $3.34 \pm 0.48$ & $2.31 \pm 0.39$ & $1.02 \pm 0.14$ & $2.49 \pm 0.09$ & $2.27 \pm 0.33$ & Submetacentric \\
\hline 12 & $3.25 \pm 0.46$ & $2.21 \pm 0.39$ & $1.05 \pm 0.12$ & $2.43 \pm 0.11$ & $2.10 \pm 0.31$ & Submetacentric \\
\hline 13 & $3.15 \pm 0.48$ & $2.14 \pm 0.39$ & $1.01 \pm 0.13$ & $2.35 \pm 0.12$ & $2.13 \pm 0.27$ & Submetacentric \\
\hline 13 & $2.98 \pm 0.50$ & $2.07 \pm 0.40$ & $0.92 \pm 0.15$ & $2.22 \pm 0.12$ & $2.27 \pm 0.39$ & Submetacentric \\
\hline 14 & $2.84 \pm 0.43$ & $1.90 \pm 0.35$ & $0.93 \pm 0.13$ & $2.11 \pm 0.06$ & $2.06 \pm 0.34$ & Submetacentric \\
\hline 14 & $2.77 \pm 0.43$ & $1.91 \pm 0.33$ & $0.86 \pm 0.13$ & $2.07 \pm 0.07$ & $2.23 \pm 0.28$ & Submetacentric \\
\hline 15 & $2.71 \pm 0.43$ & $1.88 \pm 0.26$ & $0.83 \pm 0.22$ & $2.02 \pm 0.09$ & $2.34 \pm 0.44$ & Submetacentric \\
\hline 15 & $2.67 \pm 0.43$ & $1.79 \pm 0.30$ & $0.87 \pm 0.16$ & $1.99 \pm 0.08$ & $2.08 \pm 0.31$ & Submetacentric \\
\hline 16 & $2.55 \pm 0.43$ & $1.75 \pm 0.32$ & $0.80 \pm 0.16$ & $1.90 \pm 0.11$ & $2.24 \pm 0.43$ & Submetacentric \\
\hline 16 & $2.48 \pm 0.45$ & $1.68 \pm 0.32$ & $0.80 \pm 0.17$ & $1.84 \pm 0.18$ & $2.14 \pm 0.37$ & Submetacentric \\
\hline 17 & $6.43 \pm 1.18$ & $5.09 \pm 0.95$ & $1.34 \pm 0.28$ & $4.77 \pm 0.20$ & $3.83 \pm 0.48$ & Subtelocentric \\
\hline 17 & $5.99 \pm 0.93$ & $4.67 \pm 0.74$ & $1.31 \pm 0.20$ & $4.46 \pm 0.15$ & $3.58 \pm 0.29$ & Subtelocentric \\
\hline 18 & $2.34 \pm 0.44$ & $1.83 \pm 0.35$ & $0.51 \pm 0.10$ & $1.75 \pm 0.18$ & $3.65 \pm 0.62$ & Subtelocentric \\
\hline 18 & $2.09 \pm 0.43$ & $1.68 \pm 0.37$ & $0.41 \pm 0.09$ & $1.55 \pm 0.15$ & $4.13 \pm 0.76$ & Subtelocentric \\
\hline 19 & $2.03 \pm 0.37$ & $1.82 \pm 0.32$ & $0.21 \pm 0.06$ & $1.51 \pm 0.13$ & $9.02 \pm 1.69$ & Acrocentric \\
\hline 19 & $1.91 \pm 0.26$ & $1.70 \pm 0.23$ & $0.20 \pm 0.05$ & $1.43 \pm 0.10$ & $8.69 \pm 1.68$ & Acrocentric \\
\hline KL & 134.22 & & & & & \\
\hline
\end{tabular}

TL: total length; L: long arm length; S: short arm length; RL: relative length; r: arm ratio, KL: karyotype length.
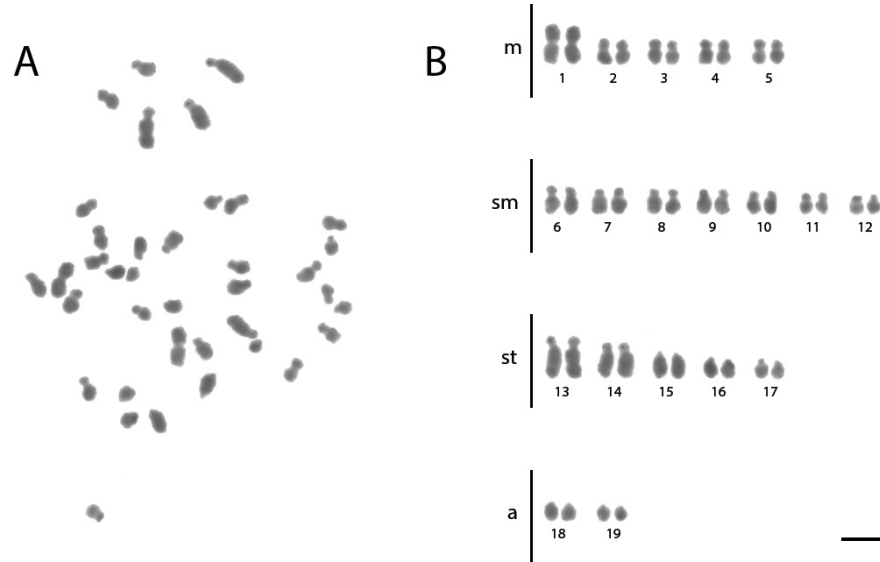

Figure 3. Conventional staining of mitotic cells of Acromyrmex lundii $\mathbf{A}$ the metaphase and $\mathbf{B}$ diploid karyotype with $2 \mathrm{n}=38$. Scale bar: $5 \mu \mathrm{m}$. 
Table 3. Karyomorphometric analyses of the chromosomes of Acromyrmex lundii.

\begin{tabular}{|c|c|c|c|c|c|c|}
\hline Chromosomes & TL & $\mathbf{L}$ & $\mathbf{S}$ & RL & $\mathbf{r}$ & Classification \\
\hline 1 & $5.00 \pm 1.17$ & $2.77 \pm 0.70$ & $2.23 \pm 0.49$ & $4.42 \pm 0.27$ & $1.24 \pm 0.11$ & Metacentric \\
\hline 1 & $4.67 \pm 1.10$ & $2.62 \pm 0.68$ & $2.05 \pm 0.44$ & $4.14 \pm 0.28$ & $1.27 \pm 0.13$ & Metacentric \\
\hline 2 & $3.16 \pm 0.63$ & $1.81 \pm 0.35$ & $1.35 \pm 0.30$ & $2.82 \pm 0.08$ & $1.35 \pm 0.11$ & Metacentric \\
\hline 2 & $3.06 \pm 0.66$ & $1.79 \pm 0.36$ & $1.27 \pm 0.32$ & $2.72 \pm 0.10$ & $1.43 \pm 0.16$ & Metacentric \\
\hline 3 & $2.91 \pm 0.61$ & $1.70 \pm 0.38$ & $1.21 \pm 0.28$ & $2.59 \pm 0.09$ & $1.42 \pm 0.21$ & Metacentric \\
\hline 3 & $2.92 \pm 0.62$ & $1.67 \pm 0.32$ & $1.25 \pm 0.32$ & $2.60 \pm 0.09$ & $1.37 \pm 0.18$ & Metacentric \\
\hline 4 & $2.81 \pm 0.53$ & $1.67 \pm 0.32$ & $1.14 \pm 0.22$ & $2.51 \pm 0.08$ & $1.47 \pm 0.11$ & Metacentric \\
\hline 4 & $2.75 \pm 0.51$ & $1.63 \pm 0.30$ & $1.13 \pm 0.21$ & $2.46 \pm 0.08$ & $1.45 \pm 0.09$ & Metacentric \\
\hline 5 & $2.61 \pm 0.43$ & $1.53 \pm 0.28$ & $1.07 \pm 0.19$ & $2.34 \pm 0.15$ & $1.44 \pm 0.18$ & Metacentric \\
\hline 5 & $2.42 \pm 0.45$ & $1.43 \pm 0.30$ & $0.99 \pm 0.16$ & $2.17 \pm 0.20$ & $1.44 \pm 0.13$ & Metacentric \\
\hline 6 & $3.38 \pm 0.65$ & $2.30 \pm 0.44$ & $1.09 \pm 0.22$ & $3.02 \pm 0.15$ & $2.12 \pm 0.22$ & Submetacentric \\
\hline 6 & $3.24 \pm 0.60$ & $2.17 \pm 0.42$ & $1.08 \pm 0.20$ & $2.89 \pm 0.11$ & $2.02 \pm 0.21$ & Submetacentric \\
\hline 7 & $3.19 \pm 0.64$ & $2.19 \pm 0.43$ & $1.00 \pm 0.22$ & $2.84 \pm 0.12$ & $2.20 \pm 0.19$ & Submetacentric \\
\hline 7 & $3.12 \pm 0.62$ & $2.19 \pm 0.48$ & $0.93 \pm 0.17$ & $2.78 \pm 0.13$ & $2.36 \pm 0.32$ & Submetacentric \\
\hline 8 & $3.02 \pm 0.55$ & $2.07 \pm 0.39$ & $0.95 \pm 0.21$ & $2.70 \pm 0.13$ & $2.21 \pm 0.36$ & Submetacentric \\
\hline 8 & $2.96 \pm 0.53$ & $2.00 \pm 0.39$ & $0.96 \pm 0.19$ & $2.65 \pm 0.12$ & $2.12 \pm 0.40$ & Submetacentric \\
\hline 9 & $2.88 \pm 0.48$ & $1.95 \pm 0.35$ & $0.94 \pm 0.17$ & $2.58 \pm 0.14$ & $2.10 \pm 0.30$ & Submetacentric \\
\hline 9 & $2.80 \pm 0.46$ & $1.89 \pm 0.32$ & $0.91 \pm 0.18$ & $2.51 \pm 0.15$ & $2.12 \pm 0.36$ & Submetacentric \\
\hline 10 & $2.70 \pm 0.50$ & $1.80 \pm 0.36$ & $0.90 \pm 0.17$ & $2.41 \pm 0.12$ & $2.02 \pm 0.28$ & Submetacentric \\
\hline 10 & $2.57 \pm 0.48$ & $1.76 \pm 0.32$ & $0.82 \pm 0.19$ & $2.30 \pm 0.13$ & $2.19 \pm 0.33$ & Submetacentric \\
\hline 11 & $2.40 \pm 0.44$ & $1.62 \pm 0.32$ & $0.78 \pm 0.14$ & $2.14 \pm 0.11$ & $2.07 \pm 0.26$ & Submetacentric \\
\hline 11 & $2.28 \pm 0.38$ & $1.58 \pm 0.34$ & $0.70 \pm 0.07$ & $2.05 \pm 0.12$ & $2.26 \pm 0.44$ & Submetacentric \\
\hline 12 & $2.18 \pm 0.32$ & $1.47 \pm 0.21$ & $0.71 \pm 0.14$ & $1.96 \pm 0.14$ & $2.10 \pm 0.26$ & Submetacentric \\
\hline 12 & $2.06 \pm 0.34$ & $1.40 \pm 0.26$ & $0.67 \pm 0.10$ & $1.85 \pm 0.13$ & $2.12 \pm 0.34$ & Submetacentric \\
\hline 13 & $5.01 \pm 1.21$ & $3.87 \pm 0.99$ & $1.14 \pm 0.24$ & $4.43 \pm 0.24$ & $3.38 \pm 0.39$ & Subtelocentric \\
\hline 13 & $4.87 \pm 1.13$ & $3.85 \pm 1.03$ & $1.02 \pm 0.12$ & $4.31 \pm 0.22$ & $3.74 \pm 0.66$ & Subtelocentric \\
\hline 14 & $4.24 \pm 0.99$ & $3.24 \pm 0.79$ & $1.00 \pm 0.20$ & $3.75 \pm 0.17$ & $3.23 \pm 0.15$ & Subtelocentric \\
\hline 14 & $4.03 \pm 1.00$ & $3.08 \pm 0.76$ & $0.95 \pm 0.24$ & $3.56 \pm 0.21$ & $3.24 \pm 0.17$ & Subtelocentric \\
\hline 15 & $3.22 \pm 0.69$ & $2.56 \pm 0.51$ & $0.66 \pm 0.20$ & $2.86 \pm 0.19$ & $4.00 \pm 0.68$ & Subtelocentric \\
\hline 15 & $3.00 \pm 0.68$ & $2.33 \pm 0.45$ & $0.66 \pm 0.25$ & $2.66 \pm 0.24$ & $3.78 \pm 0.97$ & Subtelocentric \\
\hline 16 & $2.65 \pm 0.66$ & $2.09 \pm 0.49$ & $0.56 \pm 0.18$ & $2.35 \pm 0.26$ & $3.89 \pm 0.74$ & Subtelocentric \\
\hline 16 & $2.38 \pm 0.51$ & $1.85 \pm 0.40$ & $0.53 \pm 0.12$ & $2.12 \pm 0.16$ & $3.53 \pm 0.26$ & Subtelocentric \\
\hline 17 & $2.27 \pm 0.47$ & $1.75 \pm 0.34$ & $0.53 \pm 0.14$ & $2.03 \pm 0.14$ & $3.40 \pm 0.39$ & Subtelocentric \\
\hline 17 & $2.09 \pm 0.33$ & $1.67 \pm 0.30$ & $0.42 \pm 0.08$ & $1.88 \pm 0.14$ & $4.07 \pm 0.88$ & Subtelocentric \\
\hline 18 & $2.06 \pm 0.37$ & $1.83 \pm 0.33$ & $0.23 \pm 0.04$ & $1.85 \pm 0.12$ & $7.92 \pm 0.58$ & Acrocentric \\
\hline 18 & $1.93 \pm 0.32$ & $1.70 \pm 0.29$ & $0.23 \pm 0.03$ & $1.73 \pm 0.14$ & $7.58 \pm 1.05$ & Acrocentric \\
\hline 19 & $1.75 \pm 0.29$ & $1.56 \pm 0.25$ & $0.18 \pm 0.05$ & $1.57 \pm 0.11$ & $8.76 \pm 1.38$ & Acrocentric \\
\hline 19 & $1.64 \pm 0.29$ & $1.48 \pm 0.26$ & $0.16 \pm 0.04$ & $1.47 \pm 0.07$ & $9.09 \pm 0.89$ & Acrocentric \\
\hline KL & 112.23 & & & & & \\
\hline
\end{tabular}

TL: total length; L: long arm length; S: short arm length; RL: relative length; r: arm ratio, KL: karyotype length.

A

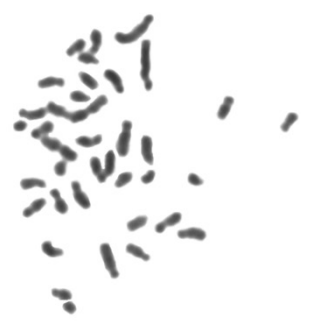

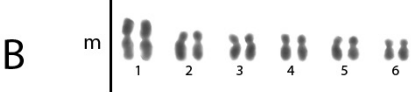

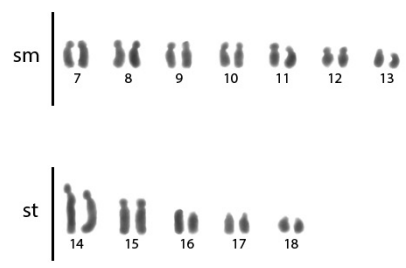

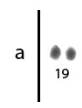

Figure 4. Conventional staining of mitotic cells of Acromyrmex nigrosetosus $\mathbf{A}$ the metaphase and $\mathbf{B}$ diploid karyotype with $2 \mathrm{n}=38$. Scale bar: $5 \mu \mathrm{m}$. 
Table 4. Karyomorphometric analyses of the chromosomes of Acromyrmex nigrosetosus.

\begin{tabular}{|c|c|c|c|c|c|c|}
\hline Chromosomes & TL & $\mathbf{L}$ & $\mathbf{S}$ & RL & $\mathbf{r}$ & Classification \\
\hline 1 & $4.40 \pm 1.10$ & $2.40 \pm 0.55$ & $2.00 \pm 0.57$ & $4.34 \pm 0.34$ & $1.22 \pm 0.11$ & Metacentric \\
\hline 1 & $4.17 \pm 1.00$ & $2.24 \pm 0.57$ & $1.93 \pm 0.44$ & $4.12 \pm 0.18$ & $1.16 \pm 0.08$ & Metacentric \\
\hline 2 & $2.92 \pm 0.61$ & $1.75 \pm 0.33$ & $1.18 \pm 0.29$ & $2.90 \pm 0.18$ & $1.51 \pm 0.12$ & Metacentric \\
\hline 2 & $2.79 \pm 0.58$ & $1.68 \pm 0.34$ & $1.12 \pm 0.24$ & $2.77 \pm 0.12$ & $1.51 \pm 0.10$ & Metacentric \\
\hline 3 & $2.71 \pm 0.54$ & $1.57 \pm 0.38$ & $1.14 \pm 0.20$ & $2.70 \pm 0.10$ & $1.38 \pm 0.21$ & Metacentric \\
\hline 3 & $2.65 \pm 0.53$ & $1.61 \pm 0.33$ & $1.06 \pm 0.22$ & $2.64 \pm 0.09$ & $1.52 \pm 0.14$ & Metacentric \\
\hline 4 & $2.59 \pm 0.53$ & $1.54 \pm 0.34$ & $1.04 \pm 0.21$ & $2.57 \pm 0.07$ & $1.48 \pm 0.13$ & Metacentric \\
\hline 4 & $2.53 \pm 0.55$ & $1.48 \pm 0.34$ & $1.05 \pm 0.22$ & $2.50 \pm 0.09$ & $1.42 \pm 0.15$ & Metacentric \\
\hline 5 & $2.44 \pm 0.55$ & $1.48 \pm 0.35$ & $0.96 \pm 0.20$ & $2.42 \pm 0.12$ & $1.54 \pm 0.11$ & Metacentric \\
\hline 5 & $2.37 \pm 0.55$ & $1.42 \pm 0.32$ & $0.96 \pm 0.24$ & $2.35 \pm 0.13$ & $1.48 \pm 0.09$ & Metacentric \\
\hline 6 & $2.24 \pm 0.56$ & $1.33 \pm 0.35$ & $0.90 \pm 0.22$ & $2.21 \pm 0.19$ & $1.48 \pm 0.12$ & Metacentric \\
\hline 6 & $2.06 \pm 0.39$ & $1.24 \pm 0.25$ & $0.82 \pm 0.14$ & $2.06 \pm 0.14$ & $1.52 \pm 0.14$ & Metacentric \\
\hline 7 & $2.99 \pm 0.55$ & $2.11 \pm 0.41$ & $0.88 \pm 0.17$ & $2.98 \pm 0.18$ & $2.42 \pm 0.27$ & Submetacentric \\
\hline 7 & $2.88 \pm 0.56$ & $2.00 \pm 0.42$ & $0.88 \pm 0.18$ & $2.87 \pm 0.14$ & $2.29 \pm 0.33$ & Submetacentric \\
\hline 8 & $2.77 \pm 0.56$ & $1.90 \pm 0.41$ & $0.87 \pm 0.18$ & $2.76 \pm 0.09$ & $2.21 \pm 0.26$ & Submetacentric \\
\hline 8 & $2.71 \pm 0.51$ & $1.87 \pm 0.32$ & $0.84 \pm 0.22$ & $2.70 \pm 0.10$ & $2.29 \pm 0.40$ & Submetacentric \\
\hline 9 & $2.69 \pm 0.52$ & $1.87 \pm 0.43$ & $0.81 \pm 0.11$ & $2.67 \pm 0.09$ & $2.30 \pm 0.36$ & Submetacentric \\
\hline 9 & $2.61 \pm 0.46$ & $1.84 \pm 0.34$ & $0.77 \pm 0.15$ & $2.60 \pm 0.13$ & $2.42 \pm 0.27$ & Submetacentric \\
\hline 10 & $2.58 \pm 0.46$ & $1.79 \pm 0.33$ & $0.79 \pm 0.16$ & $2.57 \pm 0.12$ & $2.29 \pm 0.35$ & Submetacentric \\
\hline 10 & $2.52 \pm 0.45$ & $1.72 \pm 0.35$ & $0.81 \pm 0.14$ & $2.51 \pm 0.14$ & $2.14 \pm 0.33$ & Submetacentric \\
\hline 11 & $2.43 \pm 0.47$ & $1.67 \pm 0.35$ & $0.76 \pm 0.14$ & $2.41 \pm 0.15$ & $2.20 \pm 0.29$ & Submetacentric \\
\hline 11 & $2.33 \pm 0.46$ & $1.59 \pm 0.33$ & $0.74 \pm 0.15$ & $2.31 \pm 0.13$ & $2.15 \pm 0.25$ & Submetacentric \\
\hline 12 & $2.24 \pm 0.42$ & $1.52 \pm 0.28$ & $0.71 \pm 0.17$ & $2.23 \pm 0.11$ & $2.20 \pm 0.39$ & Submetacentric \\
\hline 12 & $2.16 \pm 0.42$ & $1.45 \pm 0.32$ & $0.70 \pm 0.15$ & $2.14 \pm 0.08$ & $2.10 \pm 0.39$ & Submetacentric \\
\hline 13 & $2.04 \pm 0.40$ & $1.39 \pm 0.29$ & $0.65 \pm 0.14$ & $2.03 \pm 0.20$ & $2.19 \pm 0.41$ & Submetacentric \\
\hline 13 & $1.91 \pm 0.35$ & $1.31 \pm 0.25$ & $0.60 \pm 0.13$ & $1.91 \pm 0.20$ & $2.25 \pm 0.42$ & Submetacentric \\
\hline 14 & $4.69 \pm 1.10$ & $3.73 \pm 0.91$ & $0.97 \pm 0.20$ & $4.64 \pm 0.20$ & $3.85 \pm 0.39$ & Subtelocentric \\
\hline 14 & $4.40 \pm 0.94$ & $3.50 \pm 0.84$ & $0.90 \pm 0.14$ & $4.36 \pm 0.12$ & $3.89 \pm 0.65$ & Subtelocentric \\
\hline 15 & $3.72 \pm 0.82$ & $2.84 \pm 0.64$ & $0.88 \pm 0.19$ & $3.68 \pm 0.14$ & $3.23 \pm 0.16$ & Subtelocentric \\
\hline 15 & $3.50 \pm 0.84$ & $2.67 \pm 0.64$ & $0.83 \pm 0.20$ & $3.46 \pm 0.18$ & $3.22 \pm 0.23$ & Subtelocentric \\
\hline 16 & $2.61 \pm 0.66$ & $2.05 \pm 0.49$ & $0.57 \pm 0.19$ & $2.60 \pm 0.43$ & $3.76 \pm 0.79$ & Subtelocentric \\
\hline 16 & $2.35 \pm 0.51$ & $1.83 \pm 0.40$ & $0.51 \pm 0.13$ & $2.34 \pm 0.31$ & $3.64 \pm 0.52$ & Subtelocentric \\
\hline 17 & $2.18 \pm 0.51$ & $1.73 \pm 0.38$ & $0.45 \pm 0.14$ & $2.17 \pm 0.27$ & $3.97 \pm 0.59$ & Subtelocentric \\
\hline 17 & $2.07 \pm 0.51$ & $1.63 \pm 0.40$ & $0.44 \pm 0.12$ & $2.05 \pm 0.22$ & $3.73 \pm 0.59$ & Subtelocentric \\
\hline 18 & $1.84 \pm 0.50$ & $1.47 \pm 0.41$ & $0.36 \pm 0.11$ & $1.81 \pm 0.16$ & $4.11 \pm 0.54$ & Subtelocentric \\
\hline 18 & $1.70 \pm 0.44$ & $1.36 \pm 0.32$ & $0.34 \pm 0.12$ & $1.68 \pm 0.12$ & $4.15 \pm 0.70$ & Subtelocentric \\
\hline 19 & $1.52 \pm 0.33$ & $1.36 \pm 0.31$ & $0.16 \pm 0.03$ & $1.51 \pm 0.13$ & $8.45 \pm 1.05$ & Acrocentric \\
\hline 19 & $1.42 \pm 0.29$ & $1.27 \pm 0.26$ & $0.16 \pm 0.03$ & $1.42 \pm 0.12$ & $8.19 \pm 0.79$ & Acrocentric \\
\hline KL & 100.73 & & & & & \\
\hline
\end{tabular}

TL: total length; L: long arm length; S: short arm length; RL: relative length; r: arm ratio, KL: karyotype length.

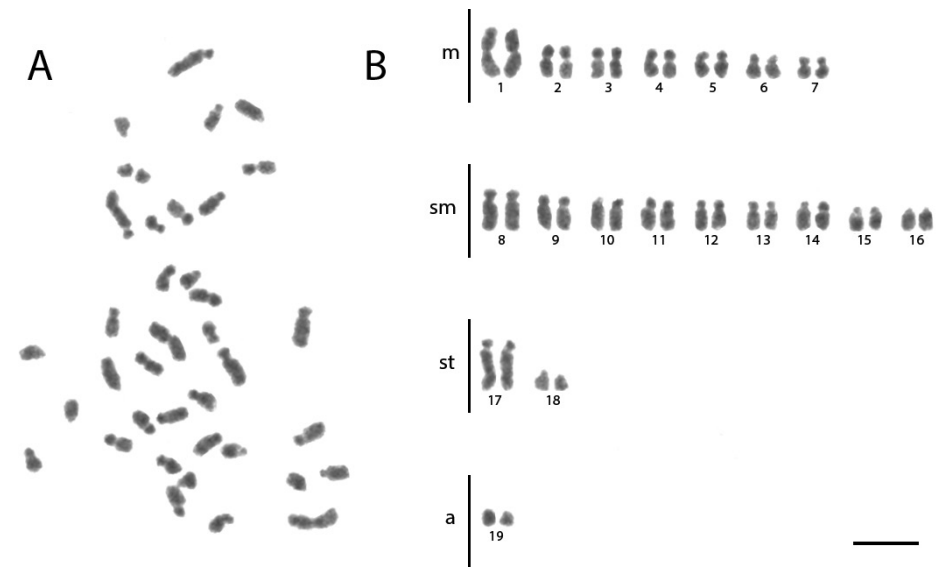

Figure 5. Conventional staining of mitotic cells of Acromyrmex subterraneus subterraneus $\mathbf{A}$ the metaphase and B diploid karyotype with $2 \mathrm{n}=38$. Scale bar: $5 \mu \mathrm{m}$. 
Table 5. Karyomorphometric analyses of the chromosomes of Acromyrmex subterraneus subterraneus.

\begin{tabular}{|c|c|c|c|c|c|c|}
\hline Chromosomes & TL & L & $S$ & RL & $r$ & Classification \\
\hline 1 & $5.03 \pm 0.96$ & $2.72 \pm 0.53$ & $2.31 \pm 0.46$ & $4.42 \pm 0.37$ & $1.19 \pm 0.11$ & Metacentric \\
\hline 1 & $4.78 \pm 0.94$ & $2.55 \pm 0.48$ & $2.23 \pm 0.48$ & $4.20 \pm 0.38$ & $1.15 \pm 0.10$ & Metacentric \\
\hline 2 & $3.31 \pm 0.64$ & $1.88 \pm 0.33$ & $1.43 \pm 0.34$ & $2.91 \pm 0.22$ & $1.34 \pm 0.18$ & Metacentric \\
\hline 2 & $3.18 \pm 0.48$ & $1.82 \pm 0.29$ & $1.37 \pm 0.21$ & $2.81 \pm 0.11$ & $1.33 \pm 0.14$ & Metacentric \\
\hline 3 & $3.08 \pm 0.45$ & $1.81 \pm 0.28$ & $1.28 \pm 0.20$ & $2.72 \pm 0.10$ & $1.42 \pm 0.15$ & Metacentric \\
\hline 3 & $3.01 \pm 0.44$ & $1.78 \pm 0.26$ & $1.23 \pm 0.20$ & $2.65 \pm 0.09$ & $1.46 \pm 0.13$ & Metacentric \\
\hline 4 & $2.96 \pm 0.46$ & $1.77 \pm 0.30$ & $1.19 \pm 0.17$ & $2.61 \pm 0.08$ & $1.49 \pm 0.11$ & Metacentric \\
\hline 4 & $2.91 \pm 0.45$ & $1.69 \pm 0.28$ & $1.22 \pm 0.18$ & $2.56 \pm 0.09$ & $1.38 \pm 0.12$ & Metacentric \\
\hline 5 & $2.87 \pm 0.45$ & $1.71 \pm 0.28$ & $1.16 \pm 0.19$ & $2.53 \pm 0.10$ & $1.48 \pm 0.13$ & Metacentric \\
\hline 5 & $2.80 \pm 0.42$ & $1.70 \pm 0.26$ & $1.10 \pm 0.17$ & $2.48 \pm 0.11$ & $1.54 \pm 0.12$ & Metacentric \\
\hline 6 & $2.70 \pm 0.42$ & $1.57 \pm 0.18$ & $1.12 \pm 0.26$ & $2.38 \pm 0.13$ & $1.45 \pm 0.21$ & Metacentric \\
\hline 6 & $2.59 \pm 0.42$ & $1.50 \pm 0.22$ & $1.09 \pm 0.24$ & $2.29 \pm 0.18$ & $1.40 \pm 0.20$ & Metacentric \\
\hline 7 & $2.46 \pm 0.38$ & $1.46 \pm 0.21$ & $1.00 \pm 0.20$ & $2.18 \pm 0.17$ & $1.48 \pm 0.17$ & Metacentric \\
\hline 7 & $2.33 \pm 0.39$ & $1.40 \pm 0.25$ & $0.93 \pm 0.15$ & $2.05 \pm 0.15$ & $1.51 \pm 0.14$ & Metacentric \\
\hline 8 & $4.35 \pm 0.99$ & $3.12 \pm 0.69$ & $1.22 \pm 0.29$ & $3.82 \pm 0.47$ & $2.56 \pm 0.26$ & Submetacentric \\
\hline 8 & $4.05 \pm 0.76$ & $2.97 \pm 0.59$ & $1.08 \pm 0.18$ & $3.56 \pm 0.32$ & $2.74 \pm 0.23$ & Submetacentric \\
\hline 9 & $3.42 \pm 0.50$ & $2.35 \pm 0.41$ & $1.08 \pm 0.17$ & $3.02 \pm 0.12$ & $2.20 \pm 0.39$ & Submetacentric \\
\hline 9 & $3.32 \pm 0.53$ & $2.29 \pm 0.44$ & $1.03 \pm 0.18$ & $2.92 \pm 0.14$ & $2.26 \pm 0.45$ & Submetacentric \\
\hline 10 & $3.23 \pm 0.53$ & $2.30 \pm 0.41$ & $0.93 \pm 0.15$ & $2.84 \pm 0.15$ & $2.49 \pm 0.34$ & Submetacentric \\
\hline 10 & $3.20 \pm 0.52$ & $2.19 \pm 0.37$ & $1.01 \pm 0.19$ & $2.82 \pm 0.15$ & $2.19 \pm 0.31$ & Submetacentric \\
\hline 11 & $3.10 \pm 0.45$ & $2.12 \pm 0.35$ & $0.98 \pm 0.15$ & $2.74 \pm 0.09$ & $2.17 \pm 0.30$ & Submetacentric \\
\hline 11 & $3.04 \pm 0.44$ & $2.11 \pm 0.33$ & $0.93 \pm 0.13$ & $2.68 \pm 0.07$ & $2.27 \pm 0.17$ & Submetacentric \\
\hline 12 & $3.01 \pm 0.44$ & $2.10 \pm 0.38$ & $0.91 \pm 0.11$ & $2.65 \pm 0.09$ & $2.31 \pm 0.36$ & Submetacentric \\
\hline 12 & $2.94 \pm 0.41$ & $2.03 \pm 0.34$ & $0.91 \pm 0.13$ & $2.60 \pm 0.10$ & $2.26 \pm 0.40$ & Submetacentric \\
\hline 13 & $2.77 \pm 0.40$ & $1.92 \pm 0.35$ & $0.84 \pm 0.11$ & $2.45 \pm 0.20$ & $2.29 \pm 0.41$ & Submetacentric \\
\hline 13 & $2.68 \pm 0.43$ & $1.85 \pm 0.33$ & $0.83 \pm 0.10$ & $2.37 \pm 0.19$ & $2.21 \pm 0.20$ & Submetacentric \\
\hline 14 & $2.58 \pm 0.38$ & $1.80 \pm 0.30$ & $0.77 \pm 0.11$ & $2.28 \pm 0.16$ & $2.34 \pm 0.30$ & Submetacentric \\
\hline 14 & $2.48 \pm 0.36$ & $1.73 \pm 0.24$ & $0.75 \pm 0.16$ & $2.20 \pm 0.17$ & $2.35 \pm 0.39$ & Submetacentric \\
\hline 15 & $2.43 \pm 0.35$ & $1.61 \pm 0.23$ & $0.82 \pm 0.16$ & $2.15 \pm 0.17$ & $2.00 \pm 0.32$ & Submetacentric \\
\hline 15 & $2.29 \pm 0.32$ & $1.60 \pm 0.26$ & $0.68 \pm 0.08$ & $2.03 \pm 0.14$ & $2.35 \pm 0.34$ & Submetacentric \\
\hline 16 & $2.23 \pm 0.30$ & $1.54 \pm 0.21$ & $0.69 \pm 0.13$ & $1.98 \pm 0.15$ & $2.26 \pm 0.35$ & Submetacentric \\
\hline 16 & $2.16 \pm 0.26$ & $1.44 \pm 0.17$ & $0.71 \pm 0.11$ & $1.91 \pm 0.11$ & $2.05 \pm 0.26$ & Submetacentric \\
\hline 17 & $4.94 \pm 0.77$ & $3.84 \pm 0.63$ & $1.1 \pm 0.17$ & $4.35 \pm 0.20$ & $3.49 \pm 0.29$ & Subtelocentric \\
\hline 17 & $4.76 \pm 0.70$ & $3.73 \pm 0.59$ & $1.03 \pm 0.15$ & $4.20 \pm 0.14$ & $3.64 \pm 0.46$ & Subtelocentric \\
\hline 18 & $2.12 \pm 0.30$ & $1.70 \pm 0.29$ & $0.42 \pm 0.05$ & $1.87 \pm 0.12$ & $4.12 \pm 0.88$ & Subtelocentric \\
\hline 18 & $1.99 \pm 0.27$ & $1.58 \pm 0.24$ & $0.41 \pm 0.08$ & $1.76 \pm 0.13$ & $4.03 \pm 1.01$ & Subtelocentric \\
\hline 19 & $1.82 \pm 0.28$ & $1.63 \pm 0.24$ & $0.19 \pm 0.04$ & $1.62 \pm 0.20$ & $8.84 \pm 1.31$ & Acrocentric \\
\hline 19 & $1.61 \pm 0.25$ & $1.46 \pm 0.21$ & $0.16 \pm 0.03$ & $1.42 \pm 0.14$ & $9.23 \pm 1.74$ & Acrocentric \\
\hline KL & 114.53 & & & & & \\
\hline
\end{tabular}

TL: total length; L: long arm length; S: short arm length; RL: relative length; r: arm ratio, KL: karyotype length.

$18 \mathrm{SM}+4 \mathrm{ST}+2 \mathrm{~A}$ (Figure 5, Table 5). For Ac. rugosus and At. sexdens only the chromosome number was established, but no detailed karyomorphometry was performed.

FISH analyses revealed that all chromosomes of all Acromyrmex species and Atta sexdens are positively marked at both arms in the telomeric regions with the presence of the canonical insect sequence (TTAGG) ${ }_{6}$ and no signals for interstitial telomeric sites were detected (Figures 6A-F, 7). The intensity and size of the probe marking was varied between the chromosomes and metaphases of each species.

\section{Discussion}

The insect canonical repeat (TTAGG) has been observed in 30 species of ants using different methods (Okazaki et al. 1993; Meyne et al. 1995; Lorite et al. 2002; Wurm 


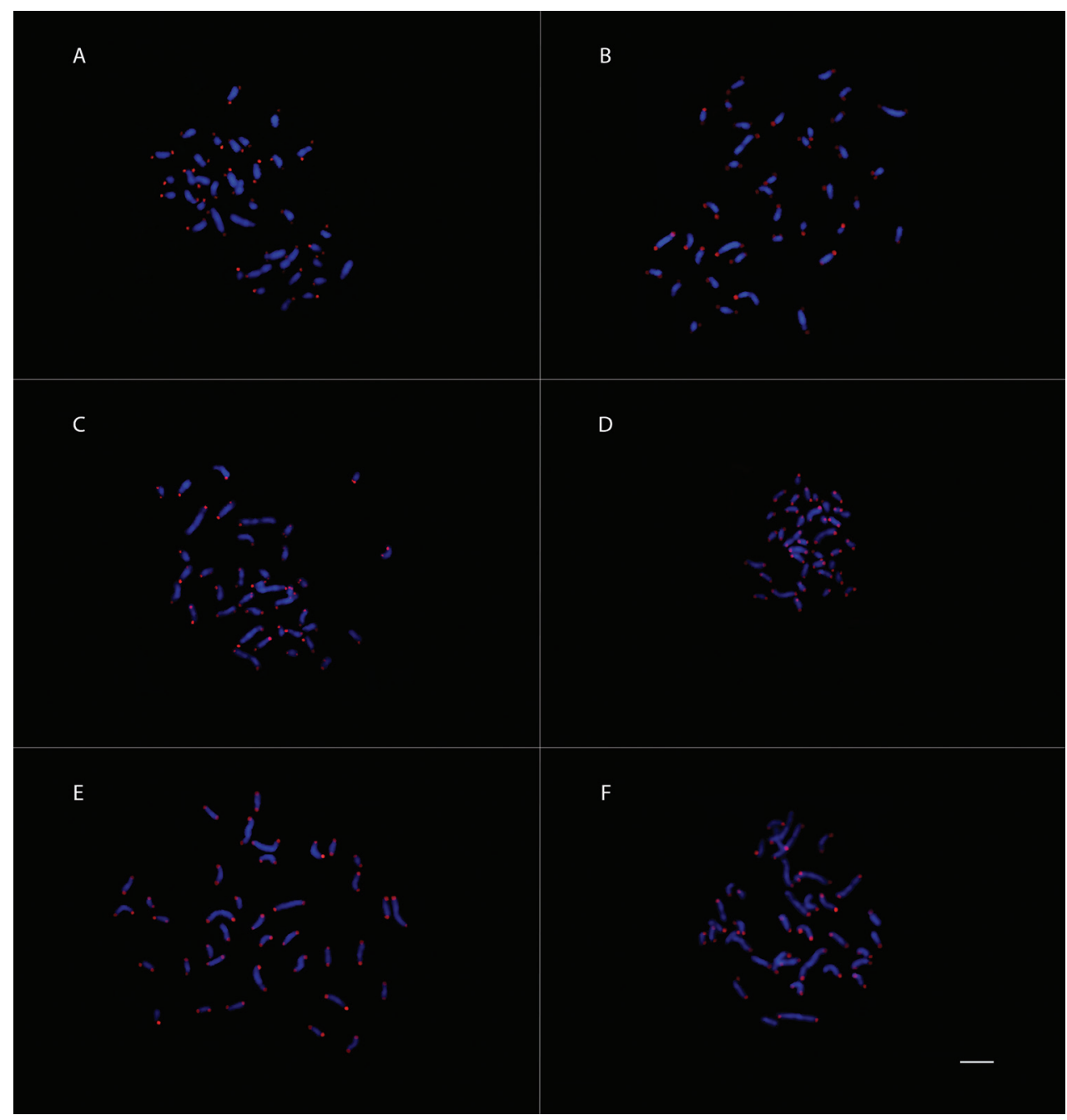

Figure 6. FISH mapping of mitotic metaphase chromosomes using a (TTAGG) ${ }_{6}$ telomeric probe; DAPI stain in blue and Cy3 in red A Acromyrmex ambiguus B Acromyrmex crassispinus $\mathbf{C}$ Acromyrmex lundii $\mathbf{D}$ Acromyrmex nigrosetosus $\mathbf{E}$ Acromyrmex rugosus and $\mathbf{F}$ Acromyrmex subterraneus subterraneus. Scale bar: $5 \mu \mathrm{m}$.

et al. 2011; Pereira et al. 2018), but FISH studies were mostly performed with Myrmecia species (Meyne et al. 1995). The only analysis involving a leafcutter ant has been performed on Ac. striatus, which also presents (TTAGG) ${ }_{6}$ labeling in the telomeres of both arms of all 22 chromosomes and does not show markings in other chromosomal regions (Pereira et al. 2018). The present study adds information about one species of Atta (At. sexdens) and six Acromyrmex species (Ac. ambiguus, Ac. crassispinus, Ac. lundii, Ac. nigrosetosus, Ac. rugosus, Ac. subterraneus subterraneus). We also describe the chromosome number and structure of Ac. lundii and Ac. nigrosetosus for the first time. The karyotype description for Ac. ambiguus from Brazil revealed the same diploid chromo- 


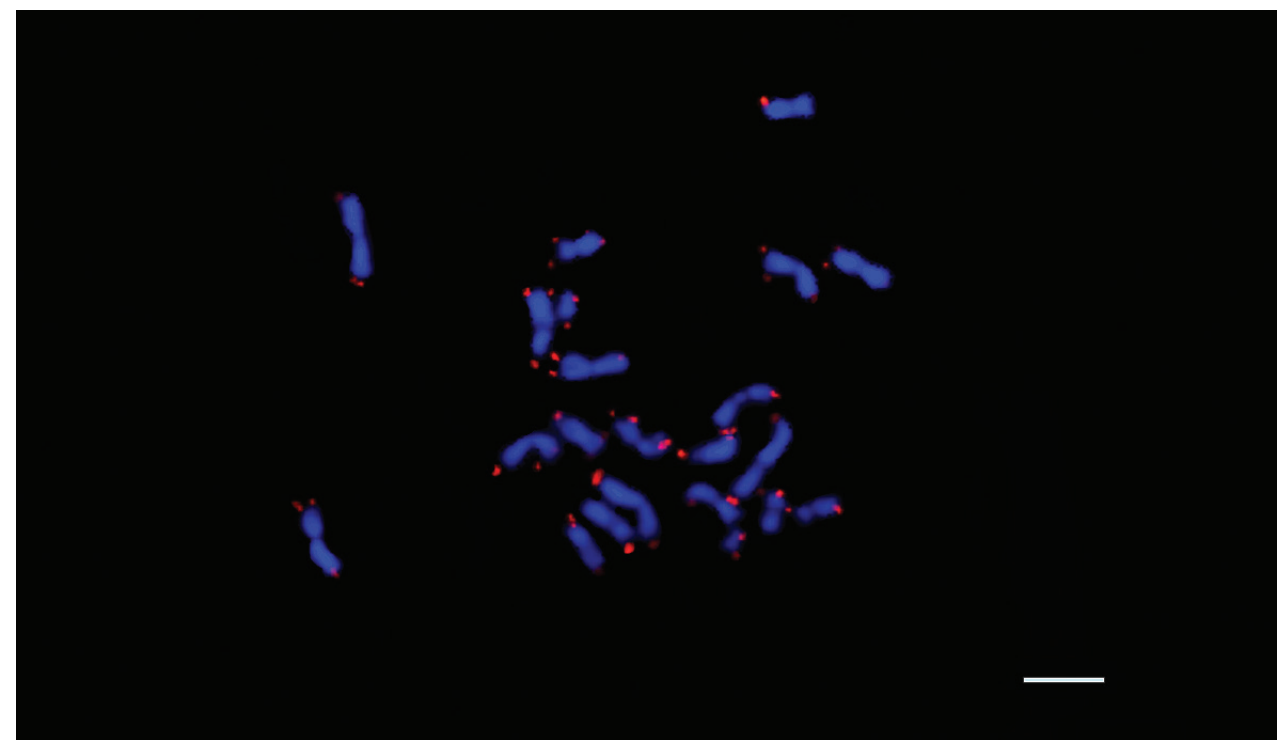

Figure 7. FISH mapping of Atta sexdens mitotic metaphase chromosomes using a (TTAGG) ${ }_{6}$ telomeric probe; DAPI in blue and Cy3 in red. Scale bar: $5 \mu \mathrm{m}$.

some number as in previous data available from Uruguay (Goñi 1983), but distinct regarding the karyotype formula, overrepresented by subtelocentric and acrocentric chromosomes in the latter. These differences may be due the visual determination of chromosome morphology instead chromosome measurements applied here. The new chromosome counts reported in this study again corroborate the stable chromosomal number in Acromyrmex and the detailed karyomorphometry of the chromosomes suggests dynamism of chromosome morphology due to distinct karyotypic formulas.

Our FISH results add to the cytogenetic knowledge of new karyotypes and molecular cytogenetic analyses in leafcutter ants, and demonstrate that the pattern found in Ac. striatus seems to occur in Atta species and Acromyrmex species. Importantly, Ac. striatus is the sister clade of Atta and the remaining Acromyrmex species (Cristiano et al. 2013). The occurrence of telomeric regions marked positively by (TTAGG) ${ }_{n}$ reinforces the premise that Formicidae presents high homology for the presence of the insect canonical sequence. This motif has been proposed to be a plesiomorphic chromosomal feature in Hymenoptera (Gokhman and Kuznetsova 2018). In fact, the canonical motif (TTAGG) ${ }_{n}$ was observed in several branches of the clade of fungus-farming ants, from anciently diverged lineages such as Mycetophylax to recent lineages such as Mycetomoellerius (Micolino et al. 2019a, b, 2020). Besides, the alternative TCAGG motif present in insects seems to be restricted to some groups, but not to Formicidae (Kuznetsova et al. 2019), and we did not find any evidences for this in previously attempted experiments in our laboratory on the phylogenetic basis of fungus-farming ants (unpublished data).

Sahara et al. (1999) propose that (TTAGG) ${ }_{n}$ is a sequence with high homology in Insecta because it is inherited from a common primitive ancestor of the class and the 
fact that some families do not show the presence of canonical repetition is explained by the group evolutionary process, where (TTAGG) ${ }_{n}$ has been lost and recovered several times. This theory is supported by Frydrychová et al. (2004) who studied 22 insect species from 20 different orders selected among the main phylogenetic group lineages and found that 15 species presented the (TTAGG) ${ }_{n}$ on their telomeres, whereas only seven species did not have the sequence in their chromosomes. The authors compared their results with the available literature and concluded that 16 insect orders have the primitive telomeric region conserved and eight do not present it. In contrast, Menezes et al. (2017) evaluated the presence of the canonical repeats (TTAGG) ${ }_{n}$ and (TTAGGG) in 25 representative species of eight Hymenoptera families, and surprisingly none of them showed any signs of these repetitive sequences in their telomeres or in any chromosomal regions. Therefore, the hypothesis regarding multiple losses of the sequence inherited from a primitive ancestor appears unlikely to these authors, as the number of Insecta families without the (TTAGG) sequence is higher than the number of those bearing it. Thus, the authors propose that the most probable evolutionary scenario is that the canonical repetition has been lost in the Apocrita ancestor or even in the Hymenoptera ancestor, whereas Apidae and Formicidae have recovered the region independently. On the contrary, the phylogenetic position and the presence of (TTAGG) ${ }_{\mathrm{n}}$ as the telomeric repeat in Tenthredo omissa (Förster, 1844) and Taxonus agrorum (Fallén, 1808) (Tenthredinidae: Symphyta) were suggested to be indicative of the ancestrality of this motif in Hymenoptera (Gokhman and Kuznetsova 2018).

Ants have high variability in their karyotypes; there are species with the haploid number of chromosomes $n=1$ (Crosland and Crozier 1986; Taylor 1991) and species with $n=60$ (Mariano et al. 2008). This variation exists with respect to the chromosome number as well as the morphology and classification. Robertsonian fissions result in two acrocentric chromosomes due to the breaking of a bi-armed chromosome, whereas Robertsonian fusions involve exactly the opposite process, where two acrocentric chromosomes unite to form a single bi-armed chromosome (Lorite and Palomeque 2010). These are possibly the two most important rearrangements for karyotype evolution in ants and support the minimum-interaction theory proposed by Imai et al. $(1988,1994,2001)$. This theory defines that fission processes are more significant and common than fusion processes because higher chromosome numbers reduce the possibility of interaction between non-homologous chromosomes within the nucleus, minimizing the mutation rates. Thus, it is proposed that the chromosomal number of ant species usually tends to increase. In this sense, it is also proposed that the ancestral karyotype of ants would be composed of a small number of metacentric chromosomes whereas recently divergent lineages would have more chromosomes due to several chromosomal fission processes (Imai et al. 1977). Thus, it is plausible to state that in Acromyrmex, karyotypes with 38 chromosomes arose following several Robertsonian fissions, whereas the chromosome number of the iconic Ac. striatus is a plesiomorphic feature maintained in Atta spp. (Cristiano et al. 2013).

Establishment of the karyotype (the chromosome number and determination of their morphology) is very important for the knowledge of chromosomal variations and possible genetic barriers between phylogenetic groups (Cristiano et al. 2017; Cardoso 
et al. 2018b). It is necessary to go further in describing the chromosome number and morphology, as more detailed karyomorphometric analyses may reveal additional and substantial variations not observed previously, mainly when accompanied with genome size estimates (Cardoso et al. 2018b). Tsutsui et al. (2008) state that closely related species, belonging to the same genus, may have very similar genome sizes, corroborating the pattern revealed by our karyomorphometric analyses in the Acromyrmex species studied here.

\section{Acknowledgement}

We are grateful to the many people that made this work possible. We thank all of our colleagues at the Lab and Research Group of Genetics and Evolution of Ants (GEFUFOP) for their help with data or their assistance in the field of chromosome preparation. The authors thank Júlio Chaul and Rodrigo Feitosa of the Universidade Federal do Paraná that confirmed the identification of the ant species. We would like to thank Editage (www.editage.com) for English language editing. We also thank the editor and two reviewers for their helpful comments on the manuscript.

\section{References}

Barros LAC, de Aguiar H, Mariano CDF, Andrade-Souza V, Costa MA, Delabie JHC, Pompolo SD (2016) Cytogenetic data on six leafcutter ants of the genus Acromyrmex Mayr, 1865 (Hymenoptera, Formicidae, Myrmicinae): insights into chromosome evolution and taxonomic implications. Comparative Cytogenetics 10(2): 229-243. https://doi.org/10.3897/ CompCytogen.v10i2.7612

Blackburn EH (1991) Structure and function of telomeres. Nature 350(6319): 569-573. https://doi.org/10.1038/350569a0

Bolton B (2020) An Online Catalog of the Ants of the World. http://antcat.org [accessed 23. March 2020]

Cardoso DC, Cristiano MP, Barros LA, Lopes DM, Pompolo SG (2012) First cytogenetic characterization of a species of the arboreal ant genus Azteca Forel, 1978 (Dolichoderinae, Formicidae). Comparative Cytogenetics 6(2): 107-114. https://doi.org/10.3897/compcytogen.v6i2.2397

Cardoso DC, Heinze J, Moura MN, Cristiano MP (2018b) Chromosomal variation among populations of a fungus-farming ant: implications for karyotype evolution and potential restriction to gene flow. BMC Evolutionary Biology 18(1): 1-146. https://doi.org/10.1186/ s12862-018-1247-5

Cardoso DC, Santos HG, Cristiano MP (2018a) The Ant Chromosome database-(ACdb): an online resource for ant (Hymenoptera: Formicidae) chromosome researchers. Myrmecological News 27: 87-91. https://doi.org/10.25849/myrmecol.news_027:087 
Cristiano MP, Cardoso DC, Fernandes-Salomão TM (2013) Cytogenetic and molecular analyses reveal a divergence between Acromyrmex striatus (Roger, 1863) and other congeneric species: taxonomic implications. PLoS ONE 8(3): e59784. https://doi.org/10.1371/journal.pone.0059784

Cristiano MP, Pereira TTP, Simóes LP, Sandoval-Gómez VE, Cardoso DC (2017) Reassessing the Chromosome Number and Morphology of the Turtle Ant Cephalotes pusillus (Klug, 1824) Using Karyomorphometrical Analysis and Observations of New Nesting Behavior. Insects 8(4): 1-114. https://doi.org/10.3390/insects8040114

Crosland MWJ, Crozier RH (1986) Myrmecia pilosula, an ant with only one pair of chromosomes. Science 231(4743): 1278-1278. https://doi.org/10.1126/science.231.4743.1278

Crozier RH (1970) Karyotypes of twenty-one ant species (Hymenoptera: Formicidae), with reviews of the known ant karyotypes. Canadian Journal of Genetics and Cytology 12(1): 109-128. https://doi.org/10.1139/g70-018

Farji-Brener AG, Ghermandi L (2008) Leaf-cutting ant nests near roads increase fitness of exotic plant species in natural protected areas. Proceedings of the Royal Society of London B 275: 1431-1440. https://doi.org/10.1098/rspb.2008.0154

Frydrychová R, Grossmann P, Trubac P, Vítková M, Marec FE (2004) Phylogenetic distribution of TTAGG telomeric repeats in insects. Genome 47(1): 163-178. https://doi. org/10.1139/g03-100

Gokhman VE, Kuznetsova VG (2018) Presence of the canonical TTAGG insect telomeric repeat in the Tenthredinidae (Symphyta) suggests its ancestral nature in the order Hymenoptera. Genetica 146(3): 341-344. https://doi.org/10.1007/s10709-018-0019-x

Goñi B, De Zolessi LC, Imai HT (1983) Karyotypes of thirteen ant species from Uruguay (Hymenoptera, Formicidae). Caryologia 36(4): 363-371. https://doi.org/10.1080/0008 7114.1983 .10797677

Imai HT, Crozier RH, Taylor RW (1977) Karyotype evolution in Australian ants. Chromosoma 59(4): 341-393. https://doi.org/10.1007/BF00327974

Imai HT, Satta Y, Takahata N (2001) Integrative study on chromosome evolution of mammals, ants and wasps based on the minimum interaction theory. Journal of Theoretical Biology 210(4): 475-497. https://doi.org/10.1006/jtbi.2001.2327

Imai HT, Taylor RW, Crozier RH (1988) Modes of spontaneous chromosomal mutation and karyotype evolution in ants with reference to the minimum interaction hypothesis. The Japanese Journal of Genetics 63(2): 159-185. https://doi.org/10.1266/jjg.63.159

Imai HT, Taylor RW, Crozier RH (1994) Experimental bases for the minimum interaction theory. I. Chromosome evolution in ants of the Myrmecia pilosula species complex (Hymenoptera: Formicidae: Myrmeciinae). The Japanese Journal of Genetics 69(2): 137-182. https://doi.org/10.1266/jjg.69.137

Kuznetsova V, Grozeva S, Gokhman V (2019) Telomere structure in insects: A review. Journal of Zoological Systematics of Evolutionary Research 58(1): 127-158. https://doi. org/10.1111/jzs.12332

Levan A, Fredga K, Sandberg A (1964) Nomenclature for centromeric position on chromosomes. Hereditas 52(2): 201-220. https://doi.org/10.1111/j.1601-5223.1964.tb01953.x 
Loeck AE, Grutzmacher D, Coimbra S, Silvana M (2003) Occurrence of leaf-cutting ants of the genus Acromyrmex in the main agricultural regions of the Rio Grande do Sul state. Current Agricultural Science and Technology 9(2): 129-133.

Lorite P, Carrillo JA, Palomeque T (2002) Conservation of (TTAGG)n telomeric sequences among ants (Hymenoptera, Formicidae). Journal of Heredity 93(4): 282-285. https://doi. org/10.1093/jhered/93.4.282

Lorite P, Palomeque T (2010) Karyotype evolution in ants (Hymenoptera: Formicidae), with a review of the known ant chromosome numbers. Myrmecological News 13: 89-102.

Mariano CDSF, Pompolo SDG, Barros LAC, Mariano-Neto E, Campiolo S, Delabie JHCA (2008) A biogeographical study of the threatened ant Dinoponera lucida Emery (Hymenoptera: Formicidae: Ponerinae) using a cytogenetic approach. Insect Conservation and Diversity 1(3): 161-168. https://doi.org/10.1111/j.1752-4598.2008.00022.x

Menezes RST, Bardella VB, Cabral de Mello DC (2017) Are the TTAGG and TTAGGG telomeric repeats phylogenetically conserved in aculeate Hymenoptera? The Science of Nature 104(9-10): 1-85. https://doi.org/10.1007/s00114-017-1507-z

Meyne J, Hirai H, Imai HT (1995) FISH analysis of the telomere sequences of bulldog ants (Myrmecia: Formicidae). Chromosoma 104(1): 14-18. https://doi.org/10.1007/ BF00352221

Micolino R, Cristiano MP, Cardoso DC (2019a) Population-Based Cytogenetic Banding Analysis and Phylogenetic Relationships of the Neotropical Fungus-Farming Ant Trachymyrmex holmgreni Wheeler, 1925. Cytogenetic and Genome Research 159(3): 151-161. https://doi.org/10.1159/000503913

Micolino R, Cristiano MP, Travenzoli NM, Lopes DM, Cardoso DC (2019b) Chromosomal dynamics in space and time: evolutionary history of Mycetophylax ants across past climatic changes in the Brazilian Atlantic coast. Scientific Reports 9(1): 1-13. https://doi. org/10.1038/s41598-019-55135-5

Micolino R, Cristiano MP, Cardoso DC (2020) Putative chromosomal inversion clue in the fungus-farming ant Mycetomoellerius iheringi Emery, 1888. Comparative Cytogenetics 14(2): 197-210. https://doi.org/10.3897/CompCytogen.v14i2.49846

Okazaki S, Tsuchida K, Maekawa H, Ishikawa H, Fujiwara H (1993) Identification of a pentanucleotide telomeric sequence, (TTAGG) n, in the silkworm Bombyx mori and in other insects. Molecular and Cellular Biology 13(3): 1424-1432. https://doi.org/10.1128/ MCB.13.3.1424

Pereira TTP, dos Reis ACCC, Cardoso DC, Cristiano MP (2018) Molecular phylogenetic reconstruction and localization of the (TTAGG)n telomeric repeats in the chromosomes of Acromyrmex striatus (Roger, 1863) suggests a lower ancestral karyotype for leafcutter ants (Hymenoptera). Comparative Cytogenetics 12(1): 13-26. https://doi.org/10.3897/CompCytogen.v12i1.21799

Sahara K, Marec F, Traut W (1999) TTAGG telomeric repeats in chromosomes of some insects and other arthropods. Chromosome Research 7(6): 449-460. https://doi. org/10.1023/A:1009297729547

Speicher MR, Carter NP (2005) The new cytogenetics: blurring the boundaries with molecular biology. Nature Reviews Genetics 6(10): 782-792. https://doi.org/10.1038/nrg1692 
Taylor RW (1991) Myrmecia croslandi sp. n., a karyologically remarkable new Australian JackJumper ant (Hymenoptera: Formicidae: Myrmeciinae). Australian Journal of Entomology 30(4): 288-288. https://doi.org/10.1111/j.1440-6055.1991.tb00438.x

Travenzoli NM, Lima BA, Cardoso DC, Dergam JA, Fernandes-Salomão TM, Lopes DM (2019) Cytogenetic analysis and chromosomal mapping of repetitive DNA in Melipona species (Hymenoptera, Meliponini). Cytogenetic and Genome Research 158(4): 213-224. https://doi.org/10.1159/000501754

Tsutsui ND, Suarez AV, Spagna JC, Johnston JS (2008) The evolution of genome size in ants. BMC Evolutionary Biology 8(1): 64-72. https://doi.org/10.1186/1471-2148-8-64

Vítková M, Král J, Traut W, Zrzavý J, Marec F (2005) The evolutionary origin of insect telomeric repeats (TTAGG)n. Chromosome Research 13(2): 145-156. https://doi.org/10.1007/ s10577-007-1910-y

Wurm Y, Wang J, Riba-Grognuz O, Corona M, Nygaard S, Brendan G. Hunt, Ingram KK, Falquet L, Nipitwattanaphon M, Gotzek D, Dijkstra MB, Oettler J, Comtesse F, Shih C-J, Wu W-J, Yang C-C, Thomas J, Beaudoing E, Pradervand S, Flegel V, Cook ED, Fabbretti R, Stockinger H, Long L, Farmerie WG, Oakey J, Boomsma JJ, Pamilo P, Yi SV, Heinze J, Goodisman MAD, Farinelli L, Harshman K, Hulo N, Cerutti L, Xenarios I, Shoemaker DW, Keller L (2011) The genome of the fire ant Solenopsis invicta. Proceedings of the National Academy of Sciences USA 108(14): 5679-5684. https://doi.org/10.1073/ pnas. 1009690108

Zakian VA (1995) Telomeres: beginning to understand the end. Science 270(5242): 16011607. https://doi.org/10.1126/science.270.5242.1601 